\title{
Consistency of Heterogeneous Multi-agent Systems Under Fixed Topology
}

\author{
Zengcheng Sun ${ }^{1, *}$, Ping $\mathrm{He}^{1,2}$, Qi Luo \\ ${ }^{1}$ Sichuan University of Science and Engineering, Huixing Road, Zigong 643000, China \\ ${ }^{2}$ Huazhong Agricultural University, Xueyuan Road, Wu Han 430070, China \\ *Correspondence Author:857217740@qq.com
}

\begin{abstract}
In this paper, we study the consistency of heterogeneous multi-intelligence systems consisting of second-order linear agent and second-order nonlinear agent under fixed topology. Firstly, the effective value range of unknown feedback gain in the control rate is determined by variable transformation method. Secondly, Lyapunov stability theory is used to make the heterogeneous multi-agent system achieve a sufficient condition of consistency under the fixed topology. Finally, a simulation example is given to verify the effectiveness and feasibility of this method.
\end{abstract}

Keywords: Heterogeneous multi-agent, Variable transformation, Consistency.

\section{Introduction}

In recent years, in order to satisfy engineering need, MIT put forward the concept of agent [1], and take the concept of individual social behavior in biology is introduced into the field of computer science. With the increasingly complex application environment, more and more scholars pay attention to the development of multi-agent system [2]-[4]. In paper [5], Jadbabaie proposes a distributed control rate, so that the first-order system based on undirected graph will eventually tend to be consistent as long as the switching topology of multi-agent system is connected. In [6], Ren proved that for the first-order system, when there is a derived tree in its communication topology, a control rate is proposed to ensure the consistency of the system. In [7], Lin Studied the connected undirected switching topology, and obtained the standard conditions for the convergence of multi-agent systems by using the method of linear transformation and constructing Lyapunov function, but this method was not extended to directed graphs and higher-order systems. In [8], Atrianfar studies the consistency problem of second-order directed topology multi-agent systems, and gives a sufficient condition. Under this condition, the state information of all agents will be close to the same value, so that the system will eventually converge. In [9], Liang studies the consistency problem of nonlinear multi-agent systems by constructing polynomial Lyapunov functions, which makes the system consistency constraints more relaxed. In [10], Zheng uses graph theory and Lyapunov stability theory to obtain the sufficient conditions for the consistency of heterogeneous systems composed of first-order and second-order agents in undirected topological graphs. In [11], Liu studied the unification of second-order nonlinear heterogeneous multi-agent system based on virtual navigator, designed the consistency protocol through Lyapunov theory, and proved its convergence. In [12], Ding using Lyapunov Krasovskii method, Ding studies the second-order nonlinear heterogeneous multi-agent consistency problem based on pilot follow method, and gives the condition that the estimation error is bounded.

The heterogeneous systems mentioned above are simply based on linear model or nonlinear model, which is obviously more meaningful for the study of heterogeneous systems including linear model and nonlinear model. In [13], Liu studies a class of heterogeneous multi-intelligent body system consistency problems consisting of a first-order linear model and a second-order Euler-Lagrangian nonlinear model and combines an adaptive controller with a PD controller to achieve consistency control given a distributed consistency protocol. In [14], Geng studied the mean consistency of firstand second-order linear intelligences and Euler-Lagrange nonlinear intelligences non-uniform systems under undirected graphs. In [15], Hou studied the consistent condition of the general second-order linear multi-agent system with communication delay, and obtained the upper bound of the tolerance delay through frequency domain analysis.

In [16], Shi targeted heterogeneous systems composed of second-order linear and second-order nonlinear intelligences (with different nonlinear terms) to make the heterogeneous systems consistent under undirected topology by Lyapunov stability theory and LaSalle invariance principle, but the method could not use heterogeneous systems composed of second-order linear and second-order nonlinear intelligences to directed graphs.

Therefore, we study the consistency problem of heterogeneous multi-intelligent questions in directed topologies based on the literature [16].

\section{Preliminaries Knowledge}

In this section, we introduce a part of the corresponding knowledge of graph theory and give the heterogeneous system model, as well as the conditions for achieving consistency.

\subsection{Graph Theory}

Suppose the graph $G=\{V, E, A\}$ is a directed weighted graph, where $V$ is the set of nodes of the directed graph $G$, and $V$ can be expressed as $V=\left\{v_{1}, v_{2} \cdots, v_{n}\right\} ; E$ denotes the set of edges between each node in graph $G$, and $E=V \times V$; $A=\left[a_{i j}\right]^{n \times n}$ denotes the adjacency matrix of graph $G$, and 
$a_{i j} \geq 0 ; i, j \in I ; I=\{1,2, \cdots, n\}$, if $\left(v_{j}, v_{i}\right) \in E$, then $a_{i j} \geq 0$; else, if $\left(v_{j}, v_{i}\right) \notin E$, then $a_{i j}=0$. Denote by $N_{i}$ the set of neighbors' agents of agent $i$. Last, The Laplacian matrix of the graph can be expressed as follows:

$$
L= \begin{cases}\sum_{j=1}^{n} a_{i j} & i=j \\ -a_{i j} & i \neq j\end{cases}
$$

Lemma 1: A directed spanning tree is a directed tree that contains all the nodes in a directed graph.

Lemma 2: A graph is said to be strongly connected when any two different nodes $v_{i}, v_{j}$ in the directed topology graph, have a mutually reachable directed path.

\subsection{System Model}

Assume a heterogeneous system consisting of $\mathrm{n}$ intelligences, where the inter-intelligence communication and state depend only on the topology formed by the directed graph $G=\{V, E, A\}$. Linear and nonlinear intelligent body systems can be represented as follows:

$$
\begin{gathered}
\left\{\begin{array}{l}
\dot{x}_{i}=v_{i} \\
\dot{v}_{i}=u_{i}
\end{array}\right. \\
\left\{\begin{array}{l}
\dot{x}_{i}=v_{i} \\
\dot{v}_{i}=u_{i}+f\left(x_{i}, v_{i}, t\right)
\end{array}\right.
\end{gathered}
$$

where $x_{i}, v_{i}, u_{i}$, denotes the displacement variable, velocity variable and control input of the $i$-th intelligent body in the intelligent body system, respectively; the nonlinear function $f\left(x_{i}, v_{i}, t\right)$ is the nonlinear term of the nonlinear intelligent body with respect to the continuous state variable.

Give consistency protocols:

$$
u_{i}=\varepsilon \sum_{j \in N_{i}} a_{i j}\left(x_{j}-x_{i}\right)-\mu v_{i} \quad i \in n
$$

Where, $\varepsilon, \mu>0$, is the consistency protocol feedback coefficient; $N_{i}$ represents the set of neighbors of agent $i$; $a_{i j}$ is the weight of adjacency matrix of agent $i$ and agent $j$.

After that, in order to design an appropriate $u_{i}$ for heterogeneous multi-agent systems to achieve consistency control objectives, the following definitions are made:

Definition 1: For both linear and nonlinear systems, given any initial conditions, there are:

$$
\left\{\begin{array}{l}
\lim _{t \rightarrow \infty}\left|x_{i}-x_{j}\right|=0 \\
\lim _{t \rightarrow \infty}\left|v_{i}-v_{j}\right|=0
\end{array}\right.
$$

This indicates that the system is consistent.

Definition 2: Assuming that the nonlinear function satisfies the Lipschitz condition, there must be a Lipschitz constant $\sigma$ such that $f\left(x_{i}, v_{i}, t\right)$ satisfies the following condition: $\left|f\left(x_{2}, v_{2}, t\right)-f\left(x_{2}, v_{2}, t\right)\right| \leq \sqrt{\sigma\left[\left(x_{2}-x_{1}\right)^{2}+\left(v_{2}-v_{1}\right)^{2}\right]}$ Where, $\forall t \geq 0,1,2$ arbitrary selections.

\section{Consistency Analysis}

In this section, the consistency protocol (3) is applied to the agent system (1) and (2) to obtain the common feedback coefficient conditions of the two parts, so as to determine the consistency protocol to achieve the consistency control goal of the heterogeneous system.

Firstly, it is assumed that the system is linear agent model, substitute consistency protocol (3) into formula (1):

$$
\left\{\begin{array}{l}
\dot{x}_{i}=v_{i} \\
\dot{v}_{i}=u_{i}=\varepsilon \sum_{j \in N_{i}} a_{i j}\left(x_{j}-x_{i}\right)-\mu v_{i}
\end{array}\right.
$$

Convert differential form to matrix form:

$$
\dot{\xi}=\left(\begin{array}{cc}
0_{n \times n} & I_{n} \\
-\varepsilon L & -\mu I_{n}
\end{array}\right) \xi
$$

Where, $\xi=\left[x^{T}, v^{T}\right]^{T}, x=\left[x_{1}, x_{2}, \cdots, x_{n}\right]^{T}, v=\left[v_{1}, v_{2}, \cdots, v_{n}\right]^{T}$.

Perform a state variable transformation, Give the matrix S:

$$
S=\left(\begin{array}{llll}
1 & 0 & \cdots & 0 \\
1 & -1 & \cdots & 0 \\
\vdots & \vdots & \ddots & \vdots \\
1 & 0 & \cdots & -1
\end{array}\right)
$$

Let $\bar{A}=S x, \bar{B}=S v$, we can get a new system matrix:

$$
\dot{\zeta}=\left(\begin{array}{cc}
0_{n \times n} & I_{n} \\
-\varepsilon S L S^{-1} & -\mu I_{n}
\end{array}\right) \zeta
$$

Where, $\zeta=\left[\bar{A}^{T}, \bar{B}^{T}\right]^{T}$.

Let's call $\zeta_{1}=\left(\bar{A}_{1}, \bar{B}_{1}\right)^{T}$, and let's call the rest $\zeta_{e}=\left(A_{2} \cdots A_{n}, B_{2} \cdots B_{n}\right)^{T}$, so system (4) can be split into the following two parts:

$$
\begin{aligned}
& \dot{\zeta}_{1}=\left[\begin{array}{cc}
0_{n-1}^{T} & 1 \\
-\varepsilon l_{1} & -\mu I_{n-1}
\end{array}\right]\left(\begin{array}{ll}
\zeta_{e 1}^{T} & \left.\bar{B}_{1}\right)^{T}
\end{array}\right. \\
& \dot{\zeta}_{e}=\left[\begin{array}{cc}
0_{(n-1) \times(n-1)} & I_{n-1} \\
-\varepsilon E L F & -\mu I_{n-1}
\end{array}\right] \zeta_{e}
\end{aligned}
$$

Where, $E=\left[1_{n-1},-I_{n-1}\right], F=\left[0_{n-1},-I_{n-1}\right]^{T}, l_{1}$ is the first row of the Laplacian matrix of communication structure graph $G$, $\bar{B}_{1}=v_{1}, \zeta_{e 1}$ represents all displacement variables in $\zeta_{e}$ except $\bar{A}_{1}$.

Similarly, the consistency protocol (3) is substituted into the nonlinear system (2), and the system is rewritten into the matrix form, which can be obtained:

$$
\dot{\bar{\xi}}=\left(\begin{array}{cc}
0_{n \times n} & I_{n} \\
-\varepsilon L & -\mu I_{n}
\end{array}\right) \bar{\xi}+\left[\begin{array}{c}
0_{n} \\
f(\bar{\xi}, t)
\end{array}\right]
$$

Where, $f(\bar{\xi}, t)=f\left(x_{1}, v_{1}, t\right), \cdots, f\left(x_{n}, v_{n}, t\right)$, and the rest of 
the symbols have the same meaning as the linear system symbols.

In the same way for nonlinear systems we can get variable transformation and split:

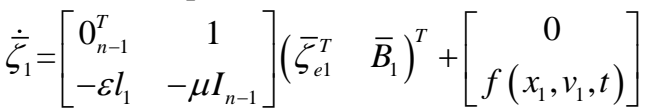

$$
\begin{aligned}
& \dot{\bar{\zeta}}_{e}=\left[\begin{array}{cc}
0_{(n-1) \times(n-1)} & I_{n-1} \\
-\varepsilon E L F & -\mu I_{n-1}
\end{array}\right] \bar{\zeta}_{e}+\left[\begin{array}{c}
0_{n-1} \\
f_{e}(\bar{\zeta}, t)
\end{array}\right]
\end{aligned}
$$

Where,

$$
f_{e}^{T}(\bar{\zeta}, t)=\left[\begin{array}{c}
f\left(\bar{A}_{1}, \bar{B}_{1}, t\right)-f\left(\bar{A}_{1}-\bar{A}_{2}, \bar{B}_{1}-\bar{B}_{2}, t\right) \\
\vdots \\
f\left(\bar{A}_{1}, \bar{B}_{1}, t\right)-f\left(\bar{A}_{1}-\bar{A}_{n}, \bar{B}_{1}-\bar{B}_{n}, t\right)
\end{array}\right],
$$

other symbols have the same meanings as those in formula (5) and (6).

Theorem 1: It is assumed that the communication topology graph $\mathrm{G}$ has a directed spanning tree. For linear systems, when the feedback coefficients $\varepsilon$ and $\mu$ of consistency protocol (3) meet the following conditions, consistency control can be achieved for linear systems.

$$
\left[\begin{array}{cc}
-\varepsilon I_{n-1} & \varepsilon(E L F)^{T} P \\
\varepsilon P(E L F) & 2(\mu-1) P
\end{array}\right]>0
$$

Theorem 2: Under the condition of theorem 1, when the feedback coefficients $\varepsilon$ and $\mu$ of consistency protocol (3) satisfy the following conditions, the nonlinear system can achieve consistency control.

$$
\left[\begin{array}{cc}
-\varepsilon I_{n-1} & \varepsilon(E L F)^{T} P \\
\varepsilon P(E L F) & 2(\mu-1) P
\end{array}\right]-I_{2} \otimes\left(2 \sigma \bar{\lambda} I_{n-1}+P\right)>0
$$

Where, $\bar{\lambda}=\lambda_{\text {max }}(P), \mathrm{P}$ is a square matrix of positive definite real numbers in $\mathrm{n}-1$ dimension, and the condition is satisfied $P(E L F)+(E L F)^{T} P=I_{n-1}, \mu>1$.

By comparing equation (10) and equation (11), it can be seen that the consistency condition of nonlinear model is more severe than that of linear model. Therefore, when the feedback coefficient of consistency protocol meets the requirement of nonlinear model, heterogeneous system can tend to be consistent.

Before proving the theorem, write down the lemma that we need.

Lemma 3: There must be a matrix $\phi$ such that two matrices $\alpha$ and $\beta$ with the same dimension satisfy the following conditions:

$$
2 \alpha^{T} \beta \leq \alpha^{T} \phi \alpha+\beta^{T} \phi^{-1} \beta
$$

Proof: given a matrix $\bar{P}=\left[\begin{array}{ll}a P & b P \\ b P & c P\end{array}\right]$

Where, $a, b, c$ satisfy $b^{2}-a c<0$.

Choose a Lyapunov function $V(t)=V_{1}(t)+V_{2}(t)$
Where, $V_{1}(t)=\zeta_{e}^{T} \bar{P} \zeta_{e}, V_{2}(t)=\bar{\zeta}_{e}^{T} \bar{P} \bar{\zeta}_{e}$.

The derivative of $V$ with respect to time $t$ :

$$
\dot{V}(t)=\dot{V}_{1}(t)+\dot{V}_{2}(t)
$$

Where:

$$
\begin{aligned}
& \dot{V}_{1}(t)=\zeta_{e}^{T}\left[\bar{P}\left(\begin{array}{cc}
0_{(n-1) \times(n-1)} & I_{n-1} \\
-\varepsilon E L F & -\mu I_{n-1}
\end{array}\right)+\left(\begin{array}{cc}
0_{(n-1) \times(n-1)} & I_{n-1} \\
-\varepsilon E L F & -\mu I_{n-1}
\end{array}\right)^{T} \bar{P}\right] \zeta_{e} \\
& =\zeta_{e}^{T}\left(\begin{array}{cc}
-\varepsilon b I_{n-1} & (a-\mu b) P-\varepsilon c(E L F)^{T} P \\
(a-\mu b) P-\varepsilon c P(E L F) & 2(b-\mu c) P
\end{array}\right) \zeta_{e}
\end{aligned}
$$

To simplify the calculation, we can choose $b=1, c=1, a=\mu$, and because $\mu>1, b^{2}-a c<0$. So the sub-function $V_{1}(t)$ is positive definite.

So $\dot{V}_{1}(t)$ can be rewritten as follows:

$$
\dot{V}_{1}(t)=-\zeta_{e}^{T}\left(\begin{array}{cc}
\varepsilon I_{n-1} & \varepsilon(E L F)^{T} P \\
\varepsilon P(E L F) & 2(\mu-1) P
\end{array}\right) \zeta_{e}
$$

According to equation (12), when $\zeta_{e} \neq 0, \dot{V}_{1}(t)<0$.

Similarly

$\dot{V}_{2}(t)=\bar{\zeta}_{e}^{T}\left[\bar{P}\left(\begin{array}{cc}0_{(n-1) \times(n-1)} & I_{n-1} \\ -\varepsilon E L F & -\mu I_{n-1}\end{array}\right)\right.$

$\left.+\left(\begin{array}{cc}0_{(n-1) \times(n-1)} & I_{n-1} \\ -\varepsilon E L F & -\mu I_{n-1}\end{array}\right)^{T} \bar{P}\right] \bar{\zeta}_{e}+2 \bar{\zeta}_{e}^{T} \bar{P}\left[\begin{array}{c}0_{n-1} \\ f_{e}(\bar{\zeta}, t)\end{array}\right]$

$=\bar{\zeta}_{e}^{T}\left(\begin{array}{cc}-\varepsilon b I_{n-1} & (a-\mu b) P-\varepsilon c(E L F)^{T} P \\ (a-\mu b) P-\varepsilon c P(E L F) & 2(b-\mu c) P\end{array}\right) \bar{\zeta}_{e}$

$+2 \bar{\zeta}_{e}^{T} \operatorname{diag}\{b P, c P\}\left[\begin{array}{l}f_{e}(\bar{\zeta}, t) \\ f_{e}(\bar{\zeta}, t)\end{array}\right]$

Similarly, choose $b=1, c=1, a=\mu, \mu>1, b^{2}-a c<0$, So can $\dot{V}_{2}(t)$ be rewritten as follows:

$$
\begin{aligned}
\dot{V}_{2}(t) & =-\bar{\zeta}_{e}^{T}\left(\begin{array}{cc}
-\varepsilon I_{n-1} & \varepsilon(E L F)^{T} P \\
\varepsilon P(E L F) & 2(\mu-1) P
\end{array}\right) \bar{\zeta}_{e} \\
& +2 \bar{\zeta}_{e}^{T} \operatorname{diag}\{b P, c P\}\left[\begin{array}{l}
f_{e}(\bar{\zeta}, t) \\
f_{e}(\bar{\zeta}, t)
\end{array}\right]
\end{aligned}
$$

According to the lemma 3:

We choose $\alpha=\bar{\zeta}_{e}, \beta=\operatorname{diag}\{P, P\}\left[\begin{array}{l}f_{e}(\bar{\zeta}, t) \\ f_{e}(\bar{\zeta}, t)\end{array}\right]$

And $\dot{V}_{2}(t)$ can be rewritten as follows:

$$
\begin{aligned}
\dot{V}_{2}(t) & \leq-\bar{\zeta}_{e}^{T}\left(\begin{array}{cc}
-\varepsilon I_{n-1} & \varepsilon(E L F)^{T} P \\
\varepsilon P(E L F) & 2(\mu-1) P
\end{array}\right) \bar{\zeta}_{e}+\bar{\zeta}_{e}^{T} \operatorname{diag}\{P, P\} \bar{\zeta}_{e} \\
& +\left[f_{e}^{T}(\bar{\zeta}, t), f_{e}^{T}(\bar{\zeta}, t)\right] \operatorname{diag}\{P, P\}\left[\begin{array}{l}
f_{e}(\bar{\zeta}, t) \\
f_{e}(\bar{\zeta}, t)
\end{array}\right]
\end{aligned}
$$

Combining equation (13) with definition 1 , equation (13) can be further rewritten as: 


$$
\begin{aligned}
\dot{V}_{2}(t) \leq-\bar{\zeta}_{e}^{T}\left(\begin{array}{cc}
-\varepsilon I_{n-1} & \varepsilon(E L F)^{T} P \\
\varepsilon P(E L F) & 2(\mu-1) P
\end{array}\right) \bar{\zeta}_{e} \\
+\bar{\zeta}_{e}^{T}\left[I_{2} \otimes\left(2 \sigma \bar{\lambda} I_{n-1}+P\right)\right] \bar{\zeta}_{e}
\end{aligned}
$$

Obvious, when $\bar{\zeta}_{e} \neq 0, \dot{V}_{2}(t)<0$.

It can be concluded that: $\dot{V}(t)=\dot{V}_{1}(t)+\dot{V}_{2}(t)<0$, it is obvious that heterogeneous systems can achieve consistency when the $\varepsilon$ and $\mu$ feedback coefficients of consistency protocol meet the conditions. To easily determine the values of $\varepsilon$ and $\mu$, we can set $\mu=\frac{1}{2} k \varepsilon+1, k>0$, and $k$ satisfy the follow condition:

$$
k P-P(E L F)(E L F)^{T} P>0
$$

The purpose of this is to realize the consistency control goal of heterogeneous system only by determining the conditions that $\varepsilon$ satisfies.

\section{Simulation Results}

The following simulation example is given. Considering the heterogeneous system composed of four agents, its communication topology is shown in Figure 1, wherein node 1 and node 4 are second-order linear agents, and node 2 and node 3 are second-order nonlinear agents.

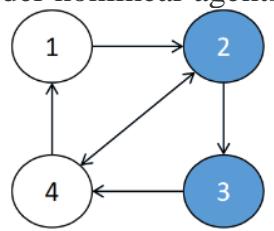

Figure 1: Communication topology of heterogeneous systems

Assume that the communication weight of the digraph is $0-1$, the Laplace matrix and E, F matrix of the system can be written from the topology diagram:

$$
\begin{gathered}
L=\left[\begin{array}{cccc}
1 & -1 & 0 & 0 \\
0 & 1 & -1 & -1 \\
0 & 0 & 1 & -1 \\
-1 & -1 & 0 & 2
\end{array}\right] \\
E=\left[\begin{array}{llll}
1 & -1 & 0 & 0 \\
1 & 0 & -1 & 0 \\
1 & 0 & 0 & -1
\end{array}\right], F=\left[\begin{array}{llll}
0 & -1 & 0 & 0 \\
0 & 0 & -1 & 0 \\
0 & 0 & 0 & -1
\end{array}\right]^{T}
\end{gathered}
$$

The calculation gives us the matrix $\mathrm{P}$ :

$$
P=\left[\begin{array}{ccc}
0.2778 & -0.0556 & 0.0214 \\
-0.0556 & 0.4444 & 0.1368 \\
0.0214 & 0.1368 & 0.3291
\end{array}\right]
$$

So, $\bar{\lambda}=\lambda_{\max }(P)=0.6977$.

When $k=2,\left|k P-P(E L F)(E L F)^{T} P\right|=0.0361>0$, we can get $\varepsilon=3 \Rightarrow \mu=4$, Choose $\sigma=2.5$, and

$\left|\left[\begin{array}{cc}-\varepsilon I_{n-1} & \varepsilon(E L F)^{T} P \\ \varepsilon P(E L F) & 2(\mu-1) P\end{array}\right]-I_{2} \otimes\left(2 \sigma \bar{\lambda} I_{n-1}+P\right)\right|=10.9303>0$
Therefore, all symbol values meet the requirements of the algorithm, continue the following analysis

The initial position and speed information of each agent are given respectively:

$$
x=[-3,3.5,6,-7.2]^{T}, v=[-1.5,5.6,-4.5,3.3]^{T}
$$

Select the nonlinear function of nodes 2 and 3:

$$
f\left(x_{i}, v_{i}, t\right)=\frac{1}{2} x_{i}(t) v_{i}(t) \sin t
$$

Therefore, the differential equation of each agent in the heterogeneous system is:

$$
\left\{\begin{array}{l}
\dot{x}_{1}(t)=v_{1}(t) \\
\dot{v}_{1}(t)=-3 x_{1}(t)+3 x_{2}(t)-4 v_{1}(t) \\
\dot{x}_{2}(t)=v_{2}(t) \\
\dot{v}_{2}(t)=-6 x_{2}(t)+3 x_{3}(t)+3 x_{4}(t)-4 v_{2}(t)+\frac{1}{2} x_{2}(t) v_{2}(t) \sin t \\
\dot{x}_{3}(t)=v_{3}(t) \\
\dot{v}_{3}(t)=-3 x_{3}(t)+3 x_{4}(t)-4 v_{3}(t)+\frac{1}{2} x_{3}(t) v_{3}(t) \sin t \\
\dot{x}_{4}(t)=v_{4}(t) \\
\dot{v}_{4}(t)=-6 x_{4}(t)+3 x_{1}(t)+3 x_{2}(t)-4 v_{4}(t)
\end{array}\right.
$$

It is assumed that the system is all composed of linear agents, and their displacement and velocity change trajectory with time is shown in Figure 2 and Figure 3.

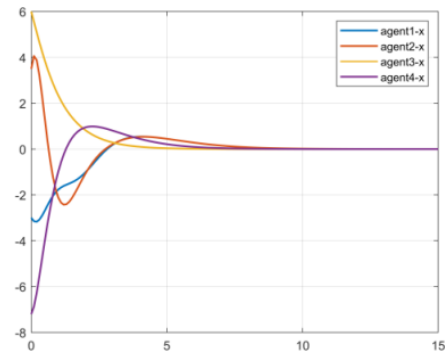

Figure 2: Linear agent displacement change

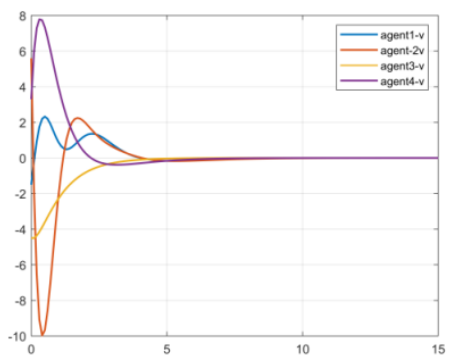

Figure 3: Linear agent speed change

Assume that the system is composed of nonlinear agents, and their displacement and velocity trajectory with time change are shown in Figure 4 and Figure 5.

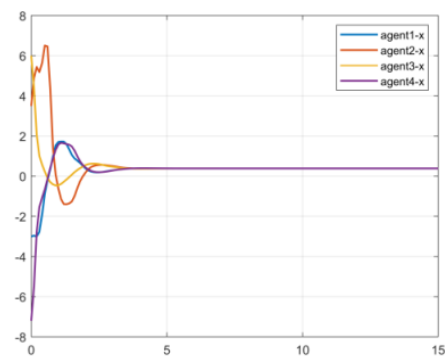

Figure 4: Nonlinear agent displacement change 


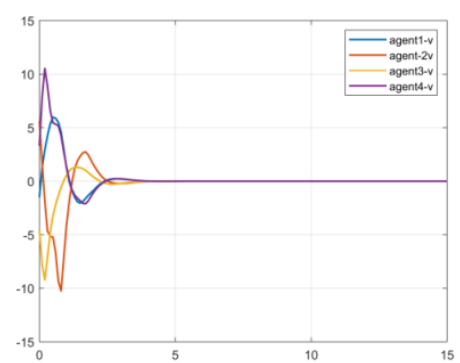

Figure 5: Nonlinear agent speed change

Displacement and velocity changes of heterogeneous multi-agent system realizing consistency control are shown in Figure 6 and Figure 7.

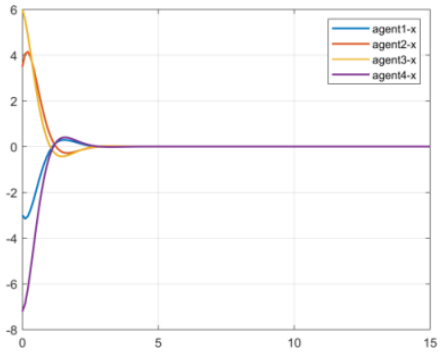

Figure 6: Heterogeneous systems displacement change

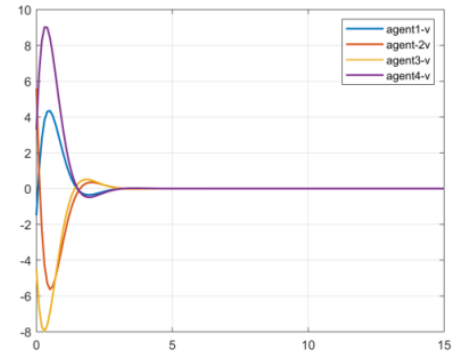

Figure 7: Heterogeneous systems speed change

As can be seen from the figure, compared with the single system, the non-uniform system accelerates the convergence speed, makes the convergence process more stable, and avoids the possibility of oscillation of nonlinear agent.

\section{Conclusion}

The consistency of heterogeneous multi-agents under any strongly connected directed fixed topology and any initial conditions is studied. The stability range of the unknown feedback gain consistency protocol is determined by Lyapunov theory and variable transformation method, and the effectiveness and feasibility of the method are verified by a simulation example. Our future work will be in the areas of higher-order systems, switching topologies and so on.

\section{References}

[1] Minsky M. Society of mind[M]. Simon and Schuster, 1988.

[2] Fax J A, Murray R M. Information flow and cooperative control of vehicle formations[J]. IEEE transactions on automatic control, 2004, 49(9): 1465-1476.

[3] Cortés J, Bullo F. Coordination and geometric optimization via distributed dynamical systems[J]. SIAM journal on control and optimization, 2005, 44(5): 1543-1574.
[4] Saber R O, Murray R M. Consensus protocols for networks of dynamic agents[J]. 2003, pp. 951-956.

[5] Jadbabaie A, Lin J, Morse A S. Coordination of groups of mobile autonomous agents using nearest neighbor rules[J]. IEEE Transactions on automatic control, 2003, 48(6): 988-1001.

[6] Ren W, Beard R W. Consensus seeking in multi-agent systems under dynamically changing interaction topologies[J]. IEEE Transactions on automatic control, 2005, 50(5): 655-661.

[7] Lin P, Jia Y. Consensus of a class of second-order multi-agent systems with time-delay and jointly-connected topologies[J]. IEEE Transactions on Automatic Control, 2010, 55(3): 778-784.

[8] Atrianfar H, Haeri M. Synchronous consensus of double-integrator continuous-time multi-agent systems with switching topologies and time-varying delays[C]. 2015 54th IEEE Conference on Decision and Control (CDC), Osaka, Japan: IEEE, 15-18 December 2015: 2205-2210.

[9] Liang Q, She Z, Wang L, General Lyapunov functions for consensus of nonlinear multi-agent systems[J]. IEEE Transactions on Circuits and Systems II: Express Briefs, 2017, 64(10): 1232-1236.

[10] Zheng Y, Zhu Y, Wang L. Consensus of heterogeneous multi-agent systems[J]. IET Control Theory \& Applications, 2011, 5(16): 1881-1888.

[11] Liu B, Wang X, Su H, Adaptive second-order consensus of multi-agent systems with heterogeneous nonlinear dynamics and time-varying delays[J]. Neurocomputing, 2013, 118: 289-300.

[12] Ding L, Zheng W X. Consensus tracking in heterogeneous nonlinear multi-agent networks with asynchronous sampled-data communication[J]. Systems \& Control Letters, 2016, 96: 151-157.

[13] Liu Y, Min H, Wang S, Distributed consensus of a class of networked heterogeneous multi-agent systems[J]. Journal of the Franklin Institute, 2014, 351(3): 1700-1716.

[14] Geng H, Chen Z, Zhang C, Consensus of heterogeneous multi-agent systems with linear and nonlinear dynamics[J]. Journal of Applied Analysis \& Computation, 2016, 6(1): 1-11.

[15] Hou W, Fu M, Zhang H, Consensus conditions for general second-order multi-agent systems with communication delay[J]. Automatica, 2017, 75: 293-298.

[16] Shi Y, Yin Y, Liu C, Consensus for heterogeneous multi-agent systems with second-order linear and nonlinear dynamics[C]. 2018 Chinese Control and Decision Conference (CCDC), Shenyang, China: IEEE, 9-11 June 2018: 6068-6071.

\section{Author Profile}

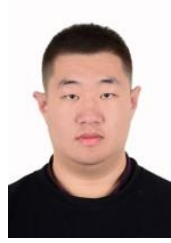

Zengcheng Sun, was born in Qingdao Shandong, China. He is Master's degree in Sichuan University of Science and Engineering 
from Zigong, China. His research direction are: multi-agent consistency and nonlinear control.

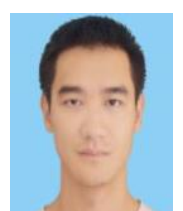

Ping He, was born in Nanchong Sichuan, China. He received doctor's degree from Hong Kong Polytechnic University. He now works at the Huazhong Agricultural University. He's main research are: multi-agent consistency, nonlinear control and robot control.

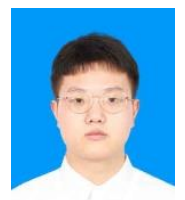

Qi Luo, was born in Guangyuan Sichuan, China. He is Master's degree in Sichuan University of Science and Engineering from Zigong, China. His research direction is tracking control for mobile robots. 